\title{
UPGRADED CALIBRATIONS OF THE THOMSON SYSTEM AT DIII-D
}

\author{
B. BRAY, C. HSIEH, T.N. CARLSTROM, and C.C. MAKARIOU
}

This is a preprint of a paper presented at the 13th Topical Conference on High-Temperature Plasma Diagnostics, June 18-22, 2000 in Tucson, Arizona and to be published in Review of Scientific Instruments.

Work supported by the U.S. Department of Energy under Contract No.

DE-AC03-99ER54463

GA PROJECT 30033

AUGUST 2000 


\section{DISCLAIMER}

This report was prepared as an account of work sponsored by an agency of the United States Government. Neither the United States Government nor any agency thereof, nor any of their employees, make any warranty, express or implied, or assumes any legal liability or responsibility for the accuracy, completeness, or usefulness of any information, apparatus, product, or process disclosed, or represents that its use would not infringe privately owned rights. Reference herein to any specific commercial product, process, or service by trade name, trademark, manufacturer, or otherwise does not necessarily constitute or imply its endorsement, recommendation, or favoring by the United States Government or any agency thereof. The views and opinions of authors expressed herein do not necessarily state or reflect those of the United States Government or any agency thereof. 


\section{DISCLAIMER}

Portions of this document may be illegible in electronic image products. Images are produced from the best available original document. 


\begin{abstract}
The DIII-D Thomson system measures electron density and temperature with eight pulsed ND:YAG lasers along three paths through the plasma vessel. The components of the Thomson system are absolutely calibrated so the measurements can be combined into a single profile from a normalized plasma radius $(\rho)$ of about 0.1 to the edge of the plasma. A monochromator calibration and opto-electronic calibration measure the detectors' absolute sensitivity to background and pulsed light. A Rayleigh scattering calibration and transmission calibrations measure the transmission of light to the detectors. The calibration systems are being upgraded to reduce the effect of systematic errors on the temperature and density measurements. The systematic errors can be checked by a comparison of overlapping channels and estimated from fits to the profiles. The contributions of the systematic uncertainties relative to the statistical uncertainties of the measurement are discussed through simulations and experimental data.
\end{abstract}




\section{INTRODUCTION}

Thomson scattering has long been a primary diagnostic for measuring the electron temperature and density in large fusion experiments. The DIII-D Thomson system ${ }^{1}$ has reliably measured $T_{e}$ from tens of $\mathrm{eV}$ to $20 \mathrm{keV}$, and $n_{e}$ from $2 \times 10^{18}$ to $2 \times 10^{20} \mathrm{~m}^{-3}$ for the past decade with significant upgrades to extend the measurements to the lower divertor ${ }^{2}$ and center of the plasma.

Measurement precision is critical when trying to compare subtle changes in the profiles from one laser pulse to the next and between plasma shots taken on different days. The system has been designed for high transmission and low noise and as a result, the Thomson system has small statistical errors.(Typically, $\delta T_{e} \sim 3 \%$ and $\delta n_{e} \sim 5 \%$ ). Recently, extensive work has begun to understand better the systematic errors in the Thomson measurement and to reduce these errors to the level of the statistical errors. 


\section{DESIGN}

The Thomson scattering system on the DIII-D Tokamak uses eight $20 \mathrm{~Hz}$ ND:YAG lasers. Light from the lasers travel along three beam paths to the core, horizontal, and divertor Thomson entry ports. ${ }^{3}$ The light is focussed in the vessel and wide angle lenses collect the scattered light from along the beam path and focus it onto an array of fiber optic cables which feed 44 polychromators.

The six wavelength channel polychromators ${ }^{4}$ were designed to maximize transmission, be compact, use $3.0 \mathrm{~cm}$ diameter optics, and be easy to align and maintain. Because 44 units are used, size and cost were factors in this design. The light is cascaded between high-performance interference filters inside the polychromators using relay lenses. The bandpass ranges of five filters were optimized to provide good temperature resolution over the range $10 \mathrm{eV}$ to $20 \mathrm{keV}$. The stray light rejection of these filters at the laser wavelength is $>10^{5}$. A $3 \mathrm{~nm}$ wide filter at the YAG wavelength is used for Rayleigh calibration. An additional low temperature filter is used for divertor polychromators to extend the temperature range for cooler plasma in the divertor regions.

Silicon avalanche photodiode detectors (APDs) are used to measure the Thomson scattered light. The APDs sensitivity to the near IR, fast response and stability make them very appropriate for detecting frequency shifted light pulses from an ND:YAG laser. The detector's gain varies about $3 \% /{ }^{\circ} \mathrm{C}$ at $1060 \mathrm{~nm}$ and they are placed away from the circuit boards next to a water-cooled polychromator frame which minimizes the temperature variation $\left(\sim 0.2^{\circ} \mathrm{C}\right)$ of the system. Highpass and direct coupled outputs from the detector measure the scattered laser light and the background light respectively. 5

The highpass and dc signals are digitized and the data is analyzed immediately after the plasma discharge with a least-squares fit to the Thomson function derived by Selden. 6 The dc signals are used to estimate the contribution of the background to the variance of the high pass signal. Typically, the total uncertainty is dominated by the high pass signal but the background 
can be significant for high power shots and channels which look toward the new upper divertor strike point. The fitter uses precalculated high pass signal levels as a function of $T_{e}$ and detector calibration to reduce the amount of computation after each plasma discharge. The profile results of $T_{e}$ and $n_{e}$ for an entire discharge are available for analysis within a minute after the end of a discharge. 


\section{CALIBRATION}

Many components of the Thomson system must be carefully calibrated to measure accurate electron temperature and density profiles. Three calibrations will be emphasized because they make significant contributions to the systematic errors of the measurements and upgrades have been made or are planned to reduce these errors.

\section{A. OPTO-ELECTRONIC CALIBRATION}

An absolutely calibrated photodiode (EG\&G Model 690) is used to measure the detector sensitivity (counts/photon) in both the high-pass and direct coupled channels by calibrating a $1060 \mathrm{~nm}$ LED (RCA C30116) operated in both pulsed and dc modes. The ratio of the gains is a function of the electronics and is therefore independent of wavelength and only the relative gain ratios of the Thomson scattering detectors to the gain ratio of the Rayleigh YAG detector is needed for profile calculation.

The calibration procedure was redesigned to eliminate the reference photodiode measurement from the final calibration used to calculate the profiles by simultaneous calibration of all detectors for an individual polychromator. The channel to channel variance of the digitizer (LeCroy FERA 4300B) for the short (65 ns) gates can be as much as a $4 \%$ correction to the observed gain ratio. The effect of the remaining $3 \%$ uncertainty in the gain ratio on the density and temperature systematic uncertainties has been modeled with a Monte Carlo simulation.

\section{B. WAVELENGTH CALIBRATION}

A monochromator (CVI Digikrom 240) is used to calibrate the wavelength response of the polychromators. This calibration is done using a dc light source and the direct coupled channel of the detectors. The ratio of high pass to dc gain corrects this calibration for the pulsed light from the lasers. Since the tranmission at the YAG wavelength is used to calibrate the measured 
density, it is expecially important to accurately calibrate the YAG filter. This includes accurately mimicing the $f / 1.75$ of the input optics because the transmission of the narrow filter (Fig. 1) is very sensitive to shifts due to the input angle of the light. The observed transmission of the YAG filter is strongly influenced by wavelength hysteresis, input angle and the $f \#$ of the input to the calibration fiber. This can introduce a $5 \%$ systematic variation in the density measurement.

To reduce the effect of this calibration on the systematic errors, a new CW ND:YAG laser has been purchased to measure the YAG transmission. This will eliminate any uncertainty due to wavelength hysteresis. A new optics system is being designed as well to better mimic the $f \#$ of the collection optics and ensure the input light is normal to the surface of the input fiber.

\section{RAYLEIGH CALIBRATION}

The YAG light filters and detectors are used to measure the Rayleigh scattering sensitivity of the polychromators. During maintenence periods, we inject Ar gas into the vessel to calibrate the Rayleigh scattering sensitivity of the polychromators. Stray laser light from the exit window and baffles which scatters off the vessel wall can be detected at significant levels on the YAG filter

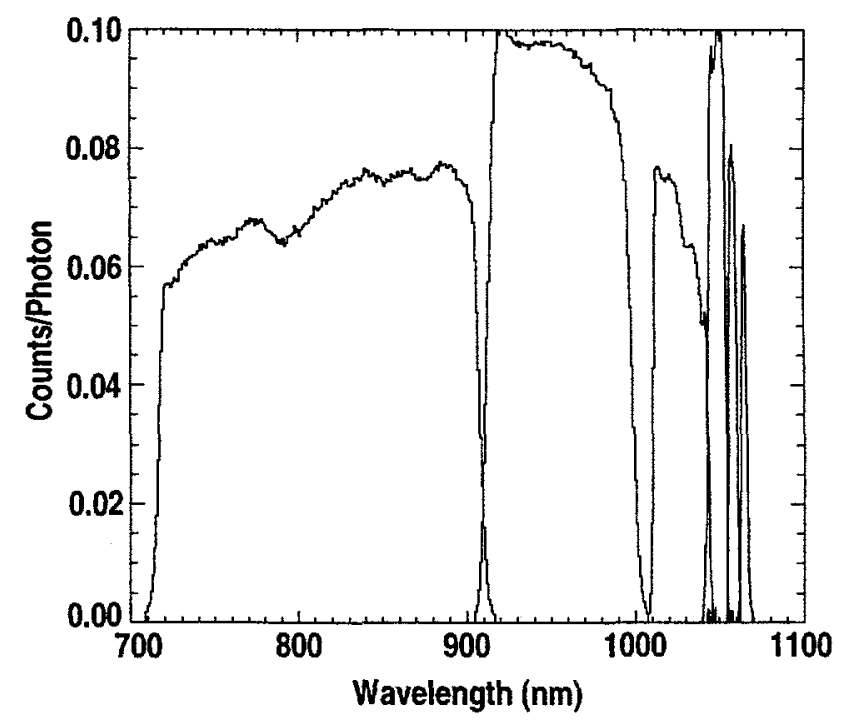

Fig. 1. Measured transmission for a six channel, Thomson scattering polychromator. 
of some of the polychromators when there is no gas in the vessel. In order to perform an accurate Rayleigh calibration, extensive effort must be devoted to reducing the level of this stray light and especially any variation in the stray light due to laser beams wandering during calibrations. Typically, a series of measurements with various pressures from 0 to 3 Torr Ar can be performed in less than one hour. During this time the position of the alignment HeNe and ND:YAG lasers must be carefully monitored.

The divertor region is expecially sensitive to the stray light because stray light from the exit window can shine directly on the divertor floor within the divertor channel viewing cones. During the 1999-2000 vent, the exit pipe was extended and exit baffles further restricted to reduce the level of stray light observed by the divertor channels by half. There is still an estimated $15 \%$ uncertainty in the divertor Rayleigh calibrations and electron density due to the variation in the stray light and alignment during calibration.

\section{LASER ALIGNMENT}

A new laser alignment feedback system for the Thomson system is being installed at DIII-D. Currently, six CCD cameras (two for each of the core, horizontal and divertor systems) are used to monitor the drift of the HeNe alignment lasers during the day. The drift is monitored and corrected manually to keep the laser system aligned. The system must be carefully aligned since a small angular drift can shift the beam significantly along its $35 \mathrm{~m}$ path.

New stepper motors have been installed in the Thomson system to allow a feedback system to correct the drift automatically. The system should be completed this year and will reduce the laser to laser variation in the Thomson density due to beam drift during plasma operations and the uncertainty in the Rayleigh calibration due to variation of the stray light level. 


\section{SYSTEMATIC ERROR COMPARISONS}

Figure 2 shows the viewing locations of the Thomson system and flux surfaces for a recent plasma discharge. The location of the spatial channels are defined by the intersection of the laser path and the viewing cone of the polychromators. Measurements are made from slightly below the midplane of the vessel to the top of the vessel and in the lower divertor. A set of closely spaced $(1.3 \mathrm{~cm})$ spatial channels view around the plasma edge region where the electron density and temperature can change rapidly.

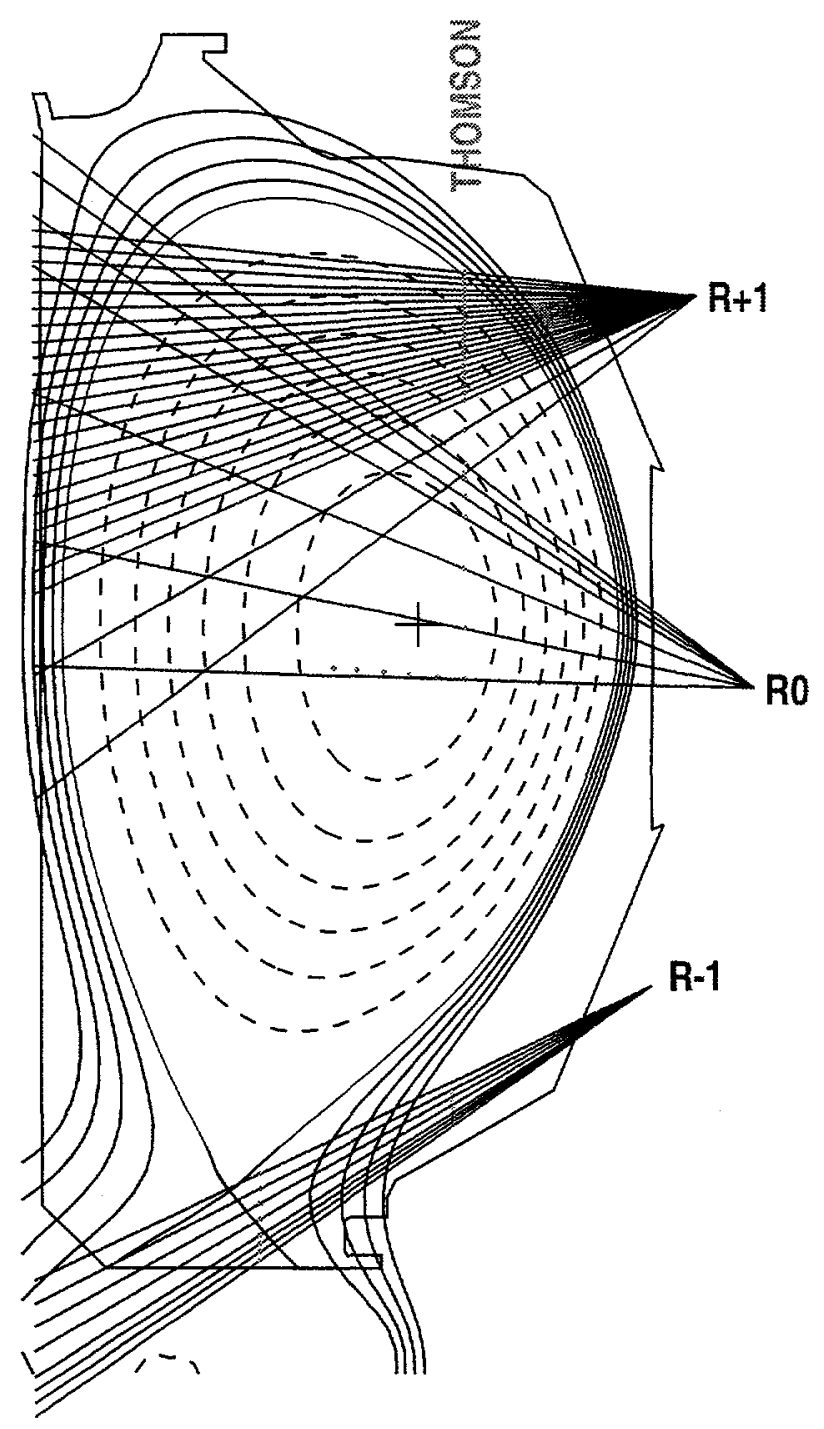

Fig. 2. Thomson viewing locations and flux surfaces for a recent lower divertor plasma discharge 
The size of the observed systematic errors can be estimated for average profiles during quiescent periods of plasma discharges. Within one second eighty profile measurements are made in the core region and systematic errors due to the calibrations can be estimated from the deviations between the average data and a fit over most $(\rho<0.8)$ of the plasma. The standard deviation of this difference is about $3 \%$ for the temperature and $6 \%$ for the density (Fig. 3). This represents a significant improvement over previous systematic errors ( $4 \%$ and $10 \%$ respectively).

Monte Carlo simulations which randomly shift the calibrations of the detectors to match the observed systematic errors suggest that the observed errors can account for most of the deviation in the average density and temperature profiles. The observed density variation is smaller than expected because recalibration of channels with large density variations has removed four points from the tails of this distribution.
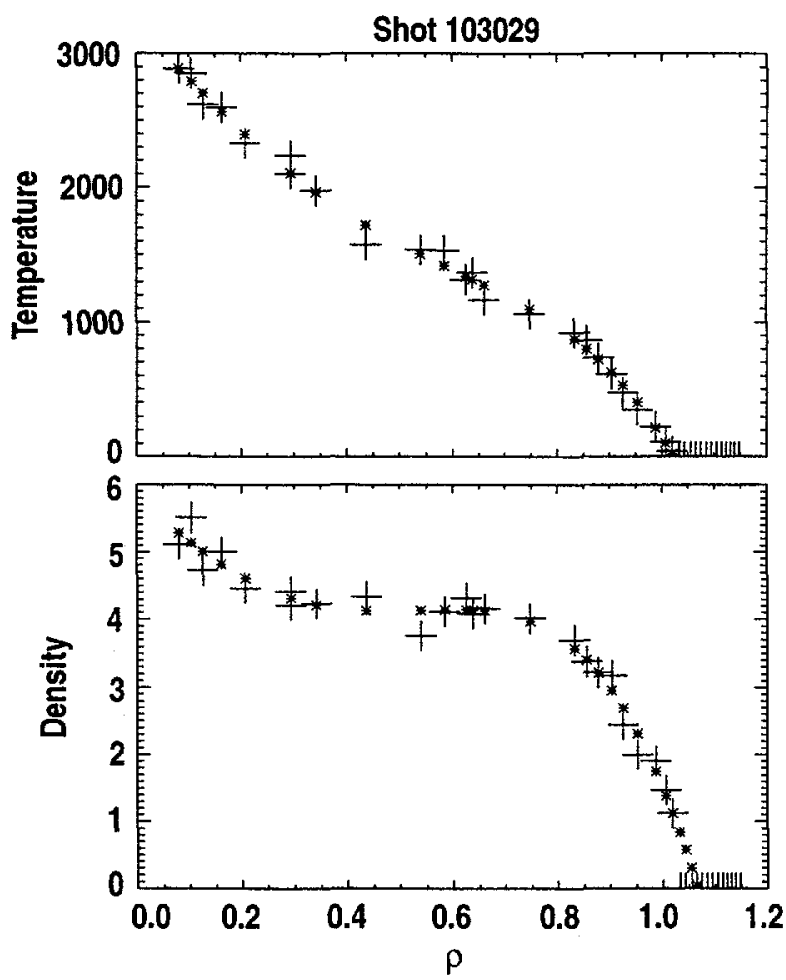

Fig. 3. Averaged Thomson profile (crosses) for a recent plasma discharge compared to a fit (circles). 
It is hoped that the proposed calibration system upgrades further diminish the systematic errors. The fitting of Thomson profiles is greatly simplified if the statistical errors are larger than the systematic errors and the improved understanding of the Thomson systematic errors increases our confidence in the measured profiles. 


\section{REFERENCES}

${ }^{1}$ T.N. Carlstrom, et al., Rev. Sci. Instr. 63(10), 4901 (1992).

2S.L. Allen, et al., J. Nucl. Mater. 241-243, 595 (1997).

3P.K. Trost, et al., Rev. Sci. Instr. 61(10), 2864 (1990).

${ }^{4}$ T.N. Carlstrom, et al., Rev. Sci. Instr. 61(10), 2858 (1990).

${ }^{5}$ C.L. Hsieh, et al., Rev. Sci. Instr. 61(10), 2855 (1990). 


\section{ACKNOWLEDGMENT}

Work supported by U.S. Department of Energy under Contract No. DE-AC03-99ER54463. 